\title{
A tomada de decisão no uso de sistema de fixação absorvível em cirurgias bucomaxilofaciais: revisão sistemática
}

Decision making in the use of absorbable fixation system in maxillofacial surgery: a systematic review Toma de decisiones en el uso de sistemas de fijación absorbibles en cirugías maxilofaciales: revisión sistemática Nilton Freitas MEDRADO FILHO

César Antonio Araújo MELO

Gilda Araújo Azevedo CUNHA

Yasmin Rebeca Santos NASCIMENTO

Georgia Costa de Araújo SOUZA

Departamento de Odontologia, Universidade do Estado do Rio Grande do Norte, UERN, 59300-000 Caicó - RN, Brasil

\section{Resumo}

Introdução: As fraturas ósseas faciais são muito comuns em lesões de face, destacando-se as fraturas mandibulares. Para o tratamento delas é necessário haver um sistema de fixação a fim de restaurar o osso fraturado, sua forma e função. Objetivo: Identificar fatores influenciadores do uso de sistemas de fixação absorvíveis em cirurgias buco maxilo faciais. Material e método: Trata-se de uma revisão sistemática feita nas bases de dados MEDLINE, BBO e SciELO, no período de agosto de 2019 a setembro de 2020 nos portais da PubMed e BVS. Dentre os 322 achados e após utilização de filtros, leitura de títulos e resumos, foram incluídos 30 artigos para compor a revisão. Resultados: Os achados do estudo revelam existir uma série de fatores influenciáveis na escolha do melhor material, tais como: sua aplicação em pacientes pediátricos ou adultos, a palpabilidade das placas, a resistência, as principais medidas de satisfação, o envolvimento com doenças malignas, complicações e novidades que a literatura vem discutindo sobre o tipo de material. Conclusão: O uso de placas absorvíveis é aconselhado para uso pediátrico, pois minimizam as restrições ao crescimento, dispensam a necessidade de uma cirurgia secundária, reduzindo, assim, gastos hospitalares. Além disso, as placas biodegradáveis originam exames de imagem com menos artefatos, de modo que não comprometem diagnósticos em pacientes com neoplasias.

Descritores: Placas Ósseas; Dispositivos de Fixação Cirúrgica; Implantes Absorvíveis.

\section{Abstract}

Introduction: Facial bone fractures are very common in facial injuries, highlighting mandibular fractures. In order to treat them, it is necessary to have a fixation system in order to restore the fractured bone, its shape and function. Objective: To identify factors influencing the use of absorbable fixation systems in oral maxillofacial surgery. Material and method: This is a systematic review carried out in the MEDLINE, BBO and SciELO databases, from August 2019 to September 2020 in the portals of PubMed and VHL. Among the 322 findings and after using filters, reading titles and abstracts, 30 articles were included to compose the review. Results: The findings of the study reveal that there are a number of influential factors in the choice of the best material, such as: its application in pediatric or adult patients, the palpability of the plates, the resistance, the main measures of satisfaction, the involvement with malignant diseases, complications and the main novelties that the literature has been discussing about the type of material. Conclusion: The use of absorbable plates is advised for pediatric use, because they minimize the restrictions to growth, dispense the need for a secondary surgery, thus reducing hospital expenses. Besides that, the biodegradable plates originate imaging exams with fewer artifacts, so that it does not compromise the diagnosis of patients with cancer.

Descriptors: Bone Plates; Surgical Fixation Devices; Absorbable Implants.

\section{Resumen}

Introducción: Las fracturas de los huesos faciales son muy frecuentes en las lesiones faciales, especialmente las fracturas mandibulares. Para poder tratarlos es necesario disponer de un sistema de fijación con el fin de restaurar el hueso fracturado, su forma y función. Objetivo: Identificar los factores que influyen en el uso de sistemas de fijación absorbible en cirugía oral maxilofacial. Material y método: Se trata de una revisión sistemática realizada en las bases de datos MEDLINE, BBO y SciELO, de agosto de 2019 a septiembre de 2020 en los portales de PubMed y VHL. Entre los 322 hallazgos y luego de utilizar filtros, leer títulos y resúmenes, se incluyeron 30 artículos para componer la revisión. Resultados: Los hallazgos del estudio revelan que existen una serie de factores que influyen en la elección del mejor material, tales como: su aplicación en pacientes pediátricos 0 adultos, la palpabilidad de las placas, la resistencia, las principales medidas de satisfacción, la implicación con enfermedades malignas, complicaciones y las principales novedades que ha venido discutiendo la literatura sobre el tipo de material. Conclusión: Se recomienda el uso de placas absorbibles para uso pediátrico, ya que minimizan las restricciones de crecimiento, prescinden de la necesidad de cirugía secundaria, reduciendo así los gastos hospitalarios. Además, las placas biodegradables dan como resultado pruebas de imagen con menos artefactos, de modo que no comprometen el diagnóstico em pacientes con neoplasias.

Descriptores: Placas Óseas; Dispositivos de Fijación Quirúrgicos; Implantes Absorbibles.

INTRODUÇÃO

Dentre as lesões mais comuns que acometem 0 esqueleto facial, podem-se destacar as fraturas mandibulares. Seu tratamento é realizado visando a restauração do osso fraturado, resultando na restauração da forma e função ${ }^{1}$. É válido ressaltar que no Brasil existe uma maior prevalência de fraturas faciais na Região Sul ${ }^{2}$.

Para superar as complicações como
estresse, identificações de corpo estranho pelo próprio organismo provenientes do uso de sistemas de fixação com placas de titânio em fraturas, foi desenvolvido um sistema de placas absorvíveis que surge como uma alternativa atraente por não necessitar de uma cirurgia secundária para remoção das placas ${ }^{3}$.

Os tipos de placas foram classificados e nomeados pela Fundação $A O$ (Arbeitsgemeinschaft für Osteosynthesefragen) desde meados de 1950 até os dias atuais, e se dividem em: metálicas, absorvíveis e de aloenxerto por osso ${ }^{4}$. As placas absorvíveis são constituídas de copolímeros tal como o ácido poliláctico (PLA) que se trata de um material osteocondutor e o ácido poliglicólico (PGA) um 
acelerador de biorreabsorção. A degradação que existe nesse tipo de material é a utilização da hidrólise do dióxido de carbono e da água presente no composto biodegradável. Além disso, a deterioração desse elemento possui a ajuda fisiológica do ciclo de Krebs ${ }^{5,6}$. A aplicação desse tipo de material ganhou bastante aceitação no manejo de pacientes acometidos por acidentes automobilísticos, sendo na maioria dos casos associados a indivíduos jovens ou por aqueles que nasceram com algum tipo de deformidade craniofacial ${ }^{7}$.

O presente trabalho objetivou identificar, na literatura nacional e internacional, fatores influenciadores do uso de sistemas de fixação absorvíveis em cirurgias bucomaxilofaciais, tendo em vista que este sistema surge como uma alternativa em contraste com os sistemas em titânio.

MATERIAL E MÉTODO

Foi conduzida uma revisão sistemática da literatura, desenvolvida seguindo as diretrizes dos itens de relatório Preferred Reporting Items for Systematic Reviews and Meta-Analyses (PRISMA) ${ }^{8}$ com modificações. A revisão sistemática tem a finalidade de reunir e sintetizar resultados de pesquisas sobre um delimitado tema ou questão, de maneira sistemática e ordenada, contribuindo para 0 aprofundamento do conhecimento do tema investigado.

- Estratégia de pesquisa e seleção de artigos

A pesquisa foi realizada nos meses de agosto de 2019 a setembro de 2020, nas bases de dados eletrônicas MEDLINE, BBO e SciELO (via Biblioteca Virtual de Saúde - BVS) e MEDLINE via PubMed, sendo a busca efetuada nos idiomas português e inglês. Como critérios de inclusão, os artigos deveriam ser estudos que auxiliassem na compreensão para a tomada de decisão no uso de sistemas de fixação com placas absorvíveis em Cirurgia e Traumatologia Buco-Maxilo-Facial. Foram utilizados os seguintes descritores localizados nos Descritores em Ciências da Saúde (DeCS): 'Placas Ósseas', 'Dispositivos de Fixação Cirúrgica' e 'Implantes Absorvíveis'. No rastreamento das publicações foram utilizados os operadores booleanos "AND" e "OR", de forma a combinar os termos/assuntos mencionados acima. A busca foi realizada por quatro pesquisadores independentes.

- Critérios de inclusão e exclusão dos estudos

A seleção dos artigos foi realizada de acordo com os termos de busca encontrados nos títulos e resumos, seguida da triagem e avaliação dos documentos completos, a fim de identificar os estudos que atenderam aos critérios de inclusão e exclusão. Portanto, foram considerados os seguintes critérios de inclusão: a) estudos dos últimos cinco anos; b) estudos realizados em humanos ou em máquinas testes; c) com texto completo e resumos disponíveis; d) deveriam ter como idiomas de publicação o inglês ou português.

Os critérios de exclusão foram artigos de metanálise, revisão sistemática, revisão de literatura, capítulos de livro, monografias e dissertações, estudos com animais (com exceção de um estudo experimental que trouxe extrema relevância ao assunto em questão), resumo de anais, duplicatas ou quaisquer outros estudos que não possuíssem relação com a área em vigor.

A seleção desses estudos foi realizada em três etapas: $1^{\mathrm{a}}$ etapa - leitura dos títulos, $2^{\mathrm{a}}$ etapa - leitura dos resumos, $3^{\underline{a}}$ etapa - leitura na íntegra dos artigos selecionados.

- Extração e análise de dados

Os dados foram extraídos manualmente e separados no software Microsoft Office Excel® 2010, no qual foram realizadas análises descritivas e quantitativas. As variáveis extraídas de cada artigo e incluídas na revisão foram: autores e ano de publicação, tipo de estudo, tamanho de amostra, material utilizado e local da fratura, assim como os principais resultados obtidos.

RESULTADOS

Dos 322 artigos obtidos na busca inicial nas bases de dados, 32 foram removidos devido ao fato de serem trabalhos duplicados. Sendo assim, os exemplares duplicados foram excluídos manualmente. De início, a seleção ocorreu por meio de leitura de título e resumo de cada artigo, obtendo-se 64 resultados e excluindo-se 23 textos que não estavam de acordo com os critérios pré-estabelecidos. Em seguida, pelos mesmos critérios, realizou-se a próxima etapa da seleção, na qual cada artigo foi lido de maneira integral e, por fim, ocorreu a composição da amostra final do presente trabalho em 30 resultados. As etapas de seleção dos estudos, bem como o número, a justificativa dos artigos excluídos e aqueles incluídos no estudo estão na Figura 1.

Os achados obtidos nesse estudo resultaram em 30 artigos, estando os anos de publicação representados na figura 2. Observase que após o ano de 2017 houve uma queda no número de publicações sobre o tema, sugerindo que a temática talvez traga um teor controverso para os cirurgiões ou mesmo que o assunto não gere tanta credibilidade, tendo em 
vista que o material em titânio ainda é considerado o padrão ouro.

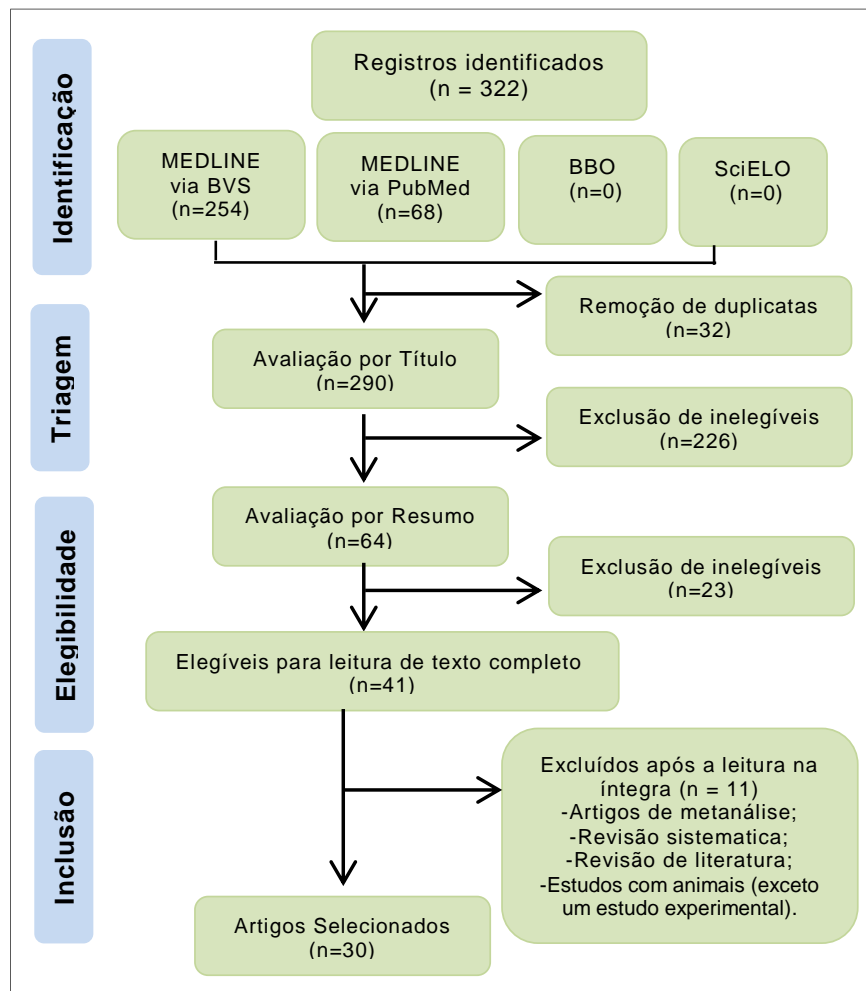

Figura 1: Fluxograma do processo de seleção dos artigos incluídos na revisão. (Fonte: Dados da pesquisa).

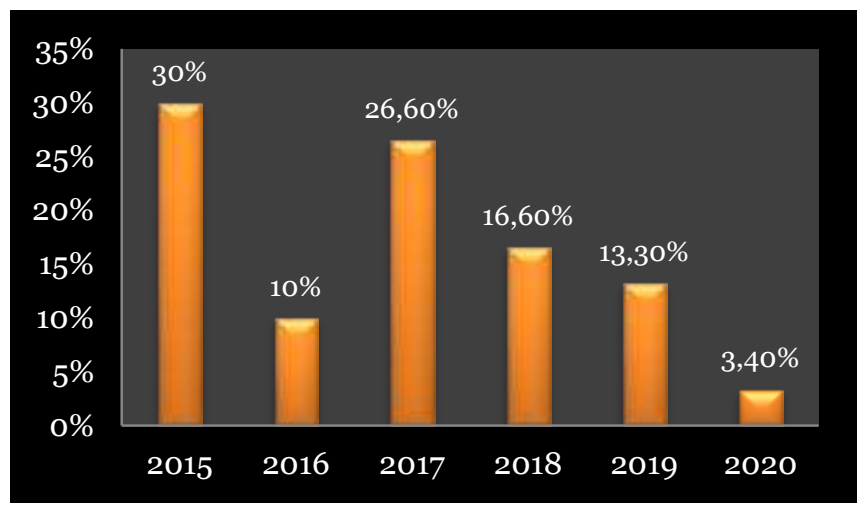

Figura 2: Porcentagem de publicações por ano no período de 2015 a 2020 das 30 publicações selecionadas. (Fonte: Dados da pesquisa).

É possível perceber que dentre todos os artigos analisados, a comparação entre dois tipos de materiais de fixação (titânio e absorvível) foi um aspecto muito comum, onde provavelmente os autores estariam em busca de qual o melhor material para os diferentes tipos de procedimentos cirúrgicos (Tabela 1) $)^{9-34}$.

Tabela 1. Distribuição dos artigos inclusos na revisão sistemática de acordo com autor/ano de publicação, tipo de estudo, tamanho da amostra, material utilizado e local da fratura (Fonte:Dados da pesquisa).

\begin{tabular}{c|c|c|c|c}
\hline Autores & $\begin{array}{c}\text { Tipo de } \\
\text { estudo }\end{array}$ & $\begin{array}{c}\text { Tamanho } \\
\text { da Amostra }\end{array}$ & $\begin{array}{c}\text { Material } \\
\text { utilizado }\end{array}$ & Local da fratura \\
\hline $\begin{array}{c}\text { Burlini et al., } \\
2015^{9}\end{array}$ & $\begin{array}{c}\text { Retrospectivo } \\
\text { observacional }\end{array}$ & 1.112 & $\begin{array}{c}\text { Reabsorvíveis ou } \\
\text { titânio }\end{array}$ & $\begin{array}{c}\text { Maxila e Mandíbula } \\
\text { (mais frequentes) }\end{array}$ \\
\hline $\begin{array}{c}\text { Yang et al., } \\
2015^{7}\end{array}$ & $\begin{array}{c}\text { Estudo } \\
\text { prospectivo }\end{array}$ & 10 & BioSorb FX 2.0 & Ângulo da mandíbula \\
\hline $\begin{array}{c}\text { Omezli et al., } \\
2015^{10}\end{array}$ & $\begin{array}{c}\text { Estudo } \\
\text { experimental }\end{array}$ & $\begin{array}{c}\text { Parafusos de } \\
\text { titânio auto- } \\
\text { roscantes } \\
\text { (Titanium Implant } \\
\text { System) e } \\
\text { parafusos } \\
\text { bioreabsorviveis } \\
\text { (Lactasorb } \\
\text { Biomet }\end{array}$ & $\begin{array}{c}\text { Fraturas condilares } \\
\text { intracapsulares }\end{array}$ & \\
\hline
\end{tabular}

Tabela 1. (Continuação) Distribuição dos artigos inclusos na revisão sistemática de acordo com autor, ano de publicação, tipo de estudo, tamanho da amostra, material utilizado e local da fratura. (Fonte: Dados da pesquisa).

\begin{tabular}{|c|c|c|c|c|}
\hline Autor/Ano & $\begin{array}{l}\text { Tipo de } \\
\text { estudo }\end{array}$ & $\begin{array}{c}\text { Tamanho } \\
\text { da Amostra }\end{array}$ & $\begin{array}{l}\text { Material } \\
\text { utilizado }\end{array}$ & Local da fratura \\
\hline $\begin{array}{l}\text { An et al., } \\
2015^{11}\end{array}$ & $\begin{array}{l}\text { Estudo } \\
\text { retrospectivo }\end{array}$ & 39 & PolyMax; Synthes & $\begin{array}{c}\text { Fraturas } \\
\text { mandibulares }\end{array}$ \\
\hline $\begin{array}{l}\text { Kim et al., } \\
2015^{12}\end{array}$ & $\begin{array}{l}\text { Retrospectivo } \\
\text { observacional }\end{array}$ & 46 & $\begin{array}{c}\text { Synthes GmbH, } \\
\text { Solothurn, Biosorb } \\
\text { FX e LINVATEC } \\
\text { Biomaterials } \\
\end{array}$ & $\begin{array}{l}\text { Defeitos segmentais } \\
\text { mandibulares }\end{array}$ \\
\hline $\begin{array}{l}\text { Rha et al., } \\
2015^{6}\end{array}$ & $\begin{array}{l}\text { Estudo } \\
\text { retrospectivo } \\
\text { clínico }\end{array}$ & 75 & $\begin{array}{l}\text { BioSorb Fx, SR- } \\
\text { PLDLA 70/30 e } \\
\text { ConMed } \\
\text { LINVATEC } \\
\end{array}$ & $\begin{array}{c}\text { Subcôndilo da } \\
\text { mandíbula, corpo da } \\
\text { mandíbula, sínfise, } \\
\text { parassínfise e ângulo } \\
\text { da mandíbula } \\
\end{array}$ \\
\hline $\begin{array}{l}\text { Sakamoto et } \\
\text { al., } 2015^{13}\end{array}$ & $\begin{array}{c}\text { Estudo } \\
\text { experimental }\end{array}$ & 3 & $\begin{array}{l}\text { LactoSorb, Biomet } \\
\mathrm{TM} \text { Micro fixation }\end{array}$ & Cranioplastia \\
\hline $\begin{array}{l}\text { OH et al., } \\
2015^{14}\end{array}$ & $\begin{array}{c}\text { Estudo } \\
\text { Experimental }\end{array}$ & 8 & $\begin{array}{l}\text { Modelo No. 8332; } \\
\text { Synbone® }\end{array}$ & $\begin{array}{c}\text { Fraturas na } \\
\text { hemimandíbula }\end{array}$ \\
\hline $\begin{array}{l}\text { Sukegawa et } \\
\text { al., 20165 }\end{array}$ & $\begin{array}{l}\text { Estudo } \\
\text { clínico }\end{array}$ & 12 & $\begin{array}{c}\text { Biorreabsorvíveis } \\
\text { mais finos } \\
\left(\text { GRAND FIX }^{\mathrm{TM}}\right) \mathrm{e} \\
\text { miniplacas de } \\
\text { titânio } \\
\text { convencionais } \\
\left.\text { (MatrixMIDFACE }^{\text {Plates }}{ }^{\mathrm{TM}}\right) \\
\end{array}$ & Fraturas zigomáticas \\
\hline $\begin{array}{l}\text { Konofaos et } \\
\text { al., 2016 }\end{array}$ & $\begin{array}{l}\text { Estudo } \\
\text { retrospectivo }\end{array}$ & 39 & $\begin{array}{c}\text { Resorb-X® mesh, } \\
\text { polyglycolic acid } \\
\text { (PGA), poly-L- } \\
\text { lactide (PLLA) } \\
\end{array}$ & Craniossinostose \\
\hline $\begin{array}{l}\text { Mahmoud et } \\
\text { al., 2016 }\end{array}$ & $\begin{array}{c}\text { Estudo } \\
\text { prospectivo }\end{array}$ & 27 & $\begin{array}{l}\text { Série BonaPlates } \\
\text { Biotech } ₫\end{array}$ & Osso zigomático \\
\hline $\begin{array}{l}\text { Leno et al., } \\
2017^{17}\end{array}$ & $\begin{array}{l}\text { Estudo } \\
\text { prospectivo }\end{array}$ & 41 & Bonaplates $\AA$ & $\begin{array}{l}\text { Corpo da mandíbula e } \\
\text { sínfise (mais } \\
\text { frequentes) }\end{array}$ \\
\hline $\begin{array}{l}\text { Essig et al., } \\
2017^{18}\end{array}$ & Relato de caso & 1 & SonicPins $\mathrm{Rx} \circledast$ & Craniossinostose \\
\hline $\begin{array}{l}\text { Nguyen et al., } \\
2017^{19}\end{array}$ & $\begin{array}{l}\text { Retrospectivo } \\
\text { observacional }\end{array}$ & 46 & Resorb-X®, Delta & Craniossinostose \\
\hline $\begin{array}{l}\text { Son et al., } \\
2017^{20}\end{array}$ & $\begin{array}{c}\text { Estudo } \\
\text { Retrospectivo }\end{array}$ & 11 & $\begin{array}{c}\text { Placas } \\
\text { biodegradáveis em } \\
\text { poli-L-lactídeo } \\
\text { (OSTEOTRANS } \\
\text { MX®) } \\
\end{array}$ & $\begin{array}{l}\text { Fratura subcondilar } \\
\text { mandibular }\end{array}$ \\
\hline $\begin{array}{c}\text { Wu et al., } \\
2018^{3}\end{array}$ & $\begin{array}{c}\text { Estudo } \\
\text { retrospectivo }\end{array}$ & 53 & Bonamates $(\AA$ & $\begin{array}{l}\text { Fratura no arco } \\
\text { zigomático }\end{array}$ \\
\hline $\begin{array}{l}\text { Chu et al., } \\
2019^{21}\end{array}$ & $\begin{array}{l}\text { Retrospectivo } \\
\text { observacional }\end{array}$ & 400 & $\begin{array}{c}\text { Inion } \AA, \\
\text { Polymax } ®, \\
\text { Osteotrans } \AA \mathrm{e} \\
\text { biosorb } \AA \\
\end{array}$ & $\begin{array}{c}\text { Complexo } \\
\text { zigomático-maxilar }\end{array}$ \\
\hline $\begin{array}{l}\text { Na et al., } \\
2019^{22}\end{array}$ & $\begin{array}{l}\text { Estudo } \\
\text { clínico }\end{array}$ & 22 & $\begin{array}{c}\text { Materiais } \\
\text { biodegradáveis }\end{array}$ & $\begin{array}{c}\text { Fraturas zigomáticas } \\
\text { unilaterais }\end{array}$ \\
\hline $\begin{array}{l}\text { Song et al., } \\
2019^{23}\end{array}$ & $\begin{array}{l}\text { Estudo de } \\
\text { coorte } \\
\text { retrospectivo }\end{array}$ & 40 & $\begin{array}{c}\text { Optimus MF } \\
\text { System } ®, \\
\text { Osteotrans MX® }\end{array}$ & Corpo da mandíbula \\
\hline $\begin{array}{l}\text { Cural et al., } \\
2018^{24}\end{array}$ & $\begin{array}{c}\text { Estudo } \\
\text { experimental }\end{array}$ & 32 & $\begin{array}{c}\text { Placas de titânio de } \\
\text { quatro furos e } \\
\text { barra de } 2 \mathrm{~mm} \\
\text { Osteosynthese, } \\
\text { placas e malhas } \\
\text { Resorb-x, } \\
\text { KLSMartin@ } \mathrm{e} \\
\text { adesivo cirúrgico } \\
\text { Omnex } 囚 \\
\end{array}$ & Ângulo mandibular \\
\hline $\begin{array}{l}\text { Ueki et al., } \\
2017^{25}\end{array}$ & $\begin{array}{c}\text { Estudo } \\
\text { prospectivo }\end{array}$ & 54 & 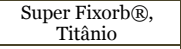 & Mandíbula \\
\hline $\begin{array}{l}\text { Janickova et } \\
\text { al., 2018 }\end{array}$ & $\begin{array}{l}\text { Estudo } \\
\text { retrospectivo }\end{array}$ & 168 & $\begin{array}{l}\text { Placas/parafusos } \\
\text { de titânio e } \\
\text { placas/parafusos } \\
\text { reabsorvíveis }\end{array}$ & $\begin{array}{c}\text { Mandíbula (corpo, } \\
\text { côndilos, } \\
\text { dentoalveolar, } \\
\text { anngulo, sínfise e } \\
\text { parassínfise), face } \\
\text { média (Le Fort, } \\
\text { complexo zigomático, } \\
\text { blow-out, naso- } \\
\text { órbito-etmoidal e } \\
\text { ossos frontais) } \\
\end{array}$ \\
\hline $\begin{array}{l}\text { Kim et al., } \\
2018^{27}\end{array}$ & $\begin{array}{l}\text { Estudo de } \\
\text { coorte } \\
\text { retrospectivo }\end{array}$ & 28 & $\begin{array}{c}\text { Placas } \\
\text { bioabsorvíveis de } \\
\text { hidroxiapatita/ } \\
\text { poli-láctideo não } \\
\text { sintetizado e } \\
\text { sistemas de } \\
\text { miniplacas de } \\
\text { titânio }\end{array}$ & $\begin{array}{l}\text { Fraturas } \\
\text { subcondilares } \\
\text { mandibulares }\end{array}$ \\
\hline $\begin{array}{l}\text { Filinte et al., } \\
2015^{28} \\
\end{array}$ & $\begin{array}{c}\text { Estudo } \\
\text { comparativo }\end{array}$ & 31 & $\begin{array}{l}\text { PLLA/PGA } \\
\text { Lactosorbß } \\
\end{array}$ & Mandíbula \\
\hline $\begin{array}{l}\text { Sukegawa et } \\
\text { al., 2018 }\end{array}$ & $\begin{array}{l}\text { Estudo } \\
\text { retrospectivo }\end{array}$ & 87 & 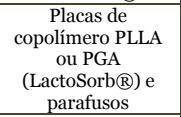 & $\begin{array}{l}\text { Região paranasal e } \\
\text { zigomático-maxilar }\end{array}$ \\
\hline $\begin{array}{l}\text { Yan et al., } \\
2019^{30}\end{array}$ & $\begin{array}{c}\text { Estudo } \\
\text { prospectivo } \\
\text { intervencional }\end{array}$ & 46 & Grand Fix & Região condilar \\
\hline 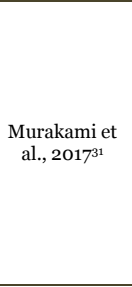 & $\begin{array}{l}\text { Estudo de caso } \\
\text { clínico }\end{array}$ & 1 & $\begin{array}{c}\text { Placa de titânio } \\
\text { com quatro } \\
\text { orifícios; Duas } \\
\text { placas retas } \\
\text { bioreabsorvíveis } \\
\text { PLLA (Super- } \\
\text { Fixsorb-MX (B); } \\
\text { Três tipos de placas } \\
\text { condilares de } \\
\text { titânio (Matrix- } \\
\text { Placas } \\
\text { Subcondilares } \\
\text { MANDÍVEIS }^{\mathrm{TM}} \text { ). } \\
\end{array}$ & Côndilo esquerdo \\
\hline $\begin{array}{l}\text { Branch et al., } \\
\qquad 2017^{32}\end{array}$ & $\begin{array}{l}\text { Estudo } \\
\text { retrospectivo }\end{array}$ & 203 & 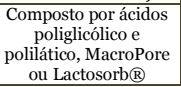 & Craniossinostose \\
\hline
\end{tabular}


Tabela 1. (Continuação) Distribuição dos artigos inclusos na revisão sistemática de acordo com autor, ano de publicação, tipo de estudo, tamanho da amostra, material utilizado e local da fratura. (Fonte: Dados da pesquisa).

\begin{tabular}{c|c|c|c|c}
\hline $\begin{array}{c}\text { Yasonov et al., } \\
2017^{33}\end{array}$ & $\begin{array}{c}\text { Estudo } \\
\text { prospectivo }\end{array}$ & 324 & $\begin{array}{c}\text { Miniplacas, Pinos e } \\
\text { gerador } \\
\text { ultrassônico Sonic } \\
\text { Weld } \AA \\
\text { Placas perfuradas } \\
\text { Barras com } \\
\text { dimensôes 50 } 5 \\
\text { mm de espessura } \\
\text { o,8 e 1 mm }\end{array}$ & Craniossinostose \\
\hline $\begin{array}{c}\text { Yan et al., } \\
2020^{34}\end{array}$ & $\begin{array}{c}\text { Estudo } \\
\text { prospectivo }\end{array}$ & 11 & $\begin{array}{c}\text { Placas } \\
\text { biorreabsorviveis }\end{array}$ & Complexo zigomático \\
\hline
\end{tabular}

DISCUSSÃO

- Uso Pediátrico

Assim como os adultos, as crianças também estão suscetíveis a sofrerem acidentes e fraturas, em consequência de práticas esportivas e/ou brincadeiras de infância. Dentre as fraturas faciais, encontram-se nos pacientes infantis principalmente as fraturas de mandíbula e de maxila. De um total de 156 casos em crianças, as maiores prevalências dos ossos envolvidos nas fraturas faciais foram: mandíbula (corpo, côndilos, dentoalveolar, ângulo, sínfise e parassínfise) em $41,7 \%$ e face média (Le Fort, complexo zigomático, blow-out, naso-órbitoetmoidal e frontal) combinada em $61,2 \%{ }^{26}$.

O trauma maxilofacial em pacientes pediátricos é particularmente desafiador, pois há impacto funcional e estético para a criança em crescimento e gera uma carga econômica e emocional para o paciente e família. Além disso, pode ser observado diferenças anatômicas e de desenvolvimento entre os pacientes pediátricos e adultos, o que pode alterar o diagnóstico e o tratamento da lesão ${ }^{35}$. A fixação interna implica uma abordagem com dissecção dos óstios, que interrompe o potencial osteogênico do periósteo e cria cicatrizes, que podem restringir ainda mais o crescimento. Microplacas e miniplacas de titânio oferecem melhoras iniciais de estabilidade, mas sua aplicação em crianças é limitada devido às preocupações sobre as restrições de crescimento, proteção contra tensões, corrosão e palpabilidade. Em contraste, a principal vantagem da fixação reabsorvível interna é a transferência gradual de carga para o osso em cicatrização durante a reabsorção, menor probabilidade de necessidade de operações secundárias para remoção do implante, bem como sem interferência ao adquirir tomografia computadorizada (TC) ou ressonância magnética $(\mathrm{RM})^{26,32}$.

Além disso, se observou através dos achados que um sistema ideal de fixação dos fragmentos ósseos não devem ser somente biocompatíveis ou rígidos para assegurar a consolidação precoce desses fragmentos, mas deve também reduzir paulatinamente sua própria rigidez, de modo a permitir estrutura óssea normal sob condições fisiológicas naturais ${ }^{33}$.

Os pacientes que tiveram fixação em metal passaram por cirurgia para retirada de 6 a 8 meses depois, enquanto 0 de material reabsorvível nenhuma cirurgia adicional foi necessária, tendo sido completamente reabsorvido dentro de 2 anos $^{9}$. Foi demonstrado que a relação custo-eficácia e a necessidade de uma cirurgia única do material reabsorvível reduzem os gastos hospitalares e melhora a qualidade de vida dos pacientes, além de minimizar a restrição ao crescimento e migração transcraniana dos dispositivos ${ }^{9,28}$. No estudo de An et. $\mathrm{al}^{11}$, foi visto uma relação de grande segurança e eficácia nesse público, sobretudo em crianças com idade média de 4 anos e 10 meses, corroborando com Filinte et al. ${ }^{28}$, que constatou em seu estudo que 31 pacientes possuíam idade média de 8 anos. Desta forma, o uso por pacientes pediátricos é bem indicado e tem sido o material de escolha para este público.

\section{- Palpabilidade das placas}

Os artigos incluídos nesta revisão também avaliaram o tempo de degradação dos materiais reabsorvíveis. Em uma comparação entre os materiais, Polymax $\AA$ e Inion $\AA$ se mostraram palpáveis em fraturas do complexo zigomático-maxilar, mesmo após 3 a 6 meses de pós-operatório, devido a sua espessura, enquanto o Osteotrans ${ }^{\circledR}$ era palpável após a cirurgia na maior parte dos pacientes, não reabsorvendo completamente mesmo após vários anos. Isso provavelmente acontece por conter hidroxiapatita. $O$ biosorb® não é palpável e tem completa reabsorção em 1 ano, mas não é tão forte quanto as outras, podendo ser usado de forma segura em fraturas do processo zigomático-maxilar isolados ${ }^{21}$.

Já na craniossinostose, estipulou-se o período de 18 meses como o tempo médio necessário para a completa reabsorção por placas utilizando a Resorb- $X^{\circledR}$ e Delta. Entre o $6^{\circ}$ e o $18^{\circ}$ mês de recuperação foi notado através do tato a formação de fluidos decorrentes da reabsorção sob o couro cabeludo dos indivíduos ${ }^{19}$. Além disso, relatouse palpabilidade em pacientes magros ${ }^{6}$.

- Resistência do material

Há uma necessidade do entendimento acerca da resistência dos materiais, visto que, trata-se de um dos fatores que irá contribuir para definição de qual material é mais adequado a ser utilizado em determinados casos. Comparações de resistência entre titânio e reabsorvíveis, indicaram semelhantes 
resultados a partir da quarta semana após o procedimento cirúrgico. Antes disso, os que usaram os reabsorvíveis tiveram menor força de mordida dos molares, além de um registro de quatro quebras do material ${ }^{17}$.

Embora não reabsorvível, o titânio é usado devido às suas excelentes características biomecânicas. No entanto, o titânio, como outros implantes metálicos, apresenta várias desvantagens, como interferência com radioterapia, produção de artefatos em tomografia computadorizada (TC) e imagem de ressonância magnética e aumento da associação com cirurgias secundárias entre os pacientes que requerem a remoção subsequente da placa após a cura da fratura ${ }^{34}$.

Portanto, placas biorreabsorvíveis, que são estáveis o suficiente para a fixação da osteossíntese e não requerem remoção posterior, foram desenvolvidas. Quanto à estabilidade, os parafusos de titânio possuem uma funcionalidade mais estável sendo comparados aos parafusos reabsorvíveis. Além disso, podemos inferir que os parafusos absorvíveis não são fortes o suficiente para uma fixação com carga maior. Isso implica que aumentar a estabilidade mecânica não garante bons resultados em detrimento de que cada indivíduo pode reagir de forma distintamente a cada produto, haja vista que o material pode ter uma fixação diferente de acordo com a propriedade feita pelos fabricantes ${ }^{10,34,36}$.

A estabilidade da fixação de pontos, sendo de 2 e 3 pontos utilizando somente materiais reabsorvíveis definiu que a fixação de 2 pontos do zigoma com materiais biodegradáveis é tão estável quanto a fixação de 3 pontos ${ }^{22,24}$, corroborando com os estudos de Ueki et al. ${ }^{25}$, e Song et al. ${ }^{23}$, onde revelaram que uma fixação híbrida de placas e parafusos absorvíveis pode ser útil e confiável em cirurgias ortognáticas e na diminuição de fraturas póscirúrgicas de corpo de mandíbula, respectivamente.

Nos estudos de Murakami et al. ${ }^{31}$ e Kim et al. ${ }^{36}$, foi constatado que é considerado difícil o fato de se colocar 4 parafusos em um fragmento condilar devido ao seu pequeno tamanho, principalmente quando se trata de casos em fratura de colo condilar. Assim, uma série de placas para fraturas condilares estão sendo projetadas em forma de trapézio, triângulo e lambda, de modo que estas sejam selecionadas a partir da particularidade de cada caso.

- Satisfação pós-operatória

Em um estudo comparativo, os pacientes que utilizaram as placas de titânio ficaram mais satisfeitos, quando comparado aos que usaram placas reabsorvíveis (Leno et al., 2017). Este resultado pode estar correlacionado com à menor força de mordida dos molares, menor extensão na abertura da boca até a quarta semana de pós-operatório pelos pacientes que usaram os reabsorvíveis, além do material reabsorvível ter sido 3 vezes mais caro que o titânio, embora não se tenha considerado os custos relativos a necessidade de uma segunda cirurgia para retirada das placas de titânio ${ }^{17}$.

Em outra literatura foi possível identificar que após a sexta semana essa satisfação também melhora no grupo do reabsorvível se assemelhando aos que usaram titânio, isso se deve a cicatrização óssea, a qual fortalece ainda mais a estabilidade da placa reabsorvível ${ }^{16}$.

Nessa perspectiva, também se relatou cura satisfatória com uma restauração favorável da forma e função, não trazendo nenhuma complicação e insatisfação por parte do paciente. Sendo assim, demonstrou-se um sucesso de tratamento, apresentando somente a palpabilidade como a única diferença presente, tendo em vista que as miniplacas de titânio e as placas absorvíveis finais possuem uma distinção na espessura ${ }^{5}$.

No estudo de Yan et al. $^{34}$, foram avaliadas medidas de satisfação antes e após a fixação com as placas biorreabsorvíveis em fraturas do complexo zigomático-maxilar (CZM), onde a restrição de abertura bucal antes do procedimento era de $72 \%$, os distúrbios neurossensoriais derivado do nervo infraorbital (90\%), diplopia (18\%) e maloclusões (54\%). Após os procedimentos cirúrgicos serem realizados com sucesso e sem complicações graves, foi constatado um excelente resultado. As oclusões dentárias foram vistas em todos os pacientes que exibiam más oclusões antes do tratamento. Os sintomas de diplopia foram resolvidos durante 0 acompanhamento, produzindo uma taxa de resolução de $100 \%$. Em relação à capacidade restrita de abrir a boca, os pacientes exibiram uma amplitude máxima de abertura bucal de mais de $3,7 \mathrm{~cm}$ durante 0 acompanhamento. Por fim, nos pacientes com dor, metade relatou diminuição da dormência, enquanto a outra metade relatou que houve resolução completa de seus sintomas, produzindo uma taxa de resolução da dor de $50 \%$.

Em contrapartida, nos estudos de Kim et al. ${ }^{36}$, ao comparar o resultado pósoperatório dos pacientes submetidos à cirurgia para inserção de placas absorvíveis com o sistema de mini placas em titânio, chegou-se à hipótese de que não há diferença de indicação 
para o uso desses dispositivos devido aos resultados serem altamente semelhantes.

\section{- Neoplasias malignas}

Segundo a literatura estudada, o sistema de placas biodegradáveis é um tipo de material indicado para indivíduos que são diagnosticados com algum tipo de ameloblastoma, osteorradionecrose, ceratocisto odontogênico, osteosarcoma, e, sobretudo o carcinoma, haja vista que o material utilizado possui absorção pelo próprio osso ${ }^{4}$. Dessa forma, os indivíduos que possuem algum processo patológico evoluídos para o câncer, como o carcinoma, podem realizar os exames através da ressonância magnética (RM) sem ocorrer sinal de alterações indesejadas ou de artefatos metálicos na $\mathrm{RM}^{36}$. A vista disso, ter 0 conhecimento desse tipo de placa é de suma importância para o cirurgião-dentista ou médico, pois ao fazer um procedimento cirúrgico terá ciência sobre de qual placa utilizar nesses indivíduos.

\section{- Complicações}

E importante frisar que todo produto ou objeto externo utilizado dentro do indivíduo tem seu aspecto positivo e negativo, dessa forma, não poderia ser diferente com os materiais reabsorvíveis. A literatura evidenciou que as complicações em função desse tipo de placa colocadas no arco do zigomático, teve como ocorrência infecções, instabilidade da fratura e reação do tecido relacionada ao implante, sendo o mais comum a existência de dor e eritema na pele do paciente ${ }^{3}$.

Portanto, essas placas ainda apresentam alguns problemas. Em primeiro lugar, dobrar placas biorreabsorvíveis é um desafio, especialmente para pessoas que são inexperientes. Em segundo lugar, há incerteza sobre se as placas biorreabsorvíveis podem gerar carga de estresse de segmentos ósseos após osteossíntese para pacientes com fraturas ${ }^{34}$. Ademais, é necessário também ter em mente que a força de resistência das placas bioabsorvíveis, em questão de uma mastigação forte, é menor quando comparada com as metálicas $^{28}$.

Além disso, as intercorrências foram observadas em fraturas da mandíbula pelo uso do implante bioabsorvível, sendo composto PLA e PGA, logo, as pequenas complicações ocorreram em função da propriedade que esses materiais possuem, provocando reação de corpo estranho, osteólise e inchaço dos tecidos moles $^{6}$. Além disso, também foi observado através da análise estatística das possíveis variáveis de pacientes com ou sem placas expostas de Ácido poli-L-lático (PLLA), que a espessura da placa foi significativamente associada ao risco de ser exposta futuramente ${ }^{29}$. Portanto, há a necessidade de rigidez suficiente para resistir às forças oclusais, pois caso contrário, pode facilmente desenvolver deiscência da ferida, levando geralmente à exposição da placa ${ }^{29}$.

Registrou-se também complicação intraoperatória, com a quebra da cabeça de parafuso. Não houve diferença estatisticamente significativa na frequência de complicações como parestesia e má oclusão ${ }^{9}$ assim como, o aparecimento de fístulas no local da incisão cirúrgica que duraram um mês, mas não houve necessidade de um tratamento especial ${ }^{17}$.

\section{- Novidades}

Embora ainda existam controvérsias em relação ao uso de fixadores reabsorvíveis no ângulo da mandíbula, a fixação com o BioSorb FX 2.0 demonstrou boa cicatrização, inexistência de complicações, ausência de fraturas de parafusos ou placas durante a cirurgia, nenhuma luxação do seguimento da fratura após a fixação das placas, bem como a presença de corpo estranho durante o acompanhamento, tendo em vista que a tomografia computadorizada auxiliou durante 6 a 12 meses nesse processo. Desse modo, em pacientes que aderem aos cuidados pósoperatórios o seu uso é totalmente indicado?.

Nesse mesmo âmbito, outra estratégia para diminuição de efeitos e complicações no uso de placas bioabsorvíveis, seria a curvatura prévia delas em um crânio impresso de forma tridimensional, contendo a fratura em questão, para então serem utilizadas na cirurgia de fato. Essa abordagem, foi posta à prova no estudo prospectivo de Yan et al. ${ }^{30}$ de fraturas do complexo zigomático-maxilar em 11 pacientes, onde houve a diminuição de aparição de segmentos de osso, maloclusão, parestesia e diplopia.

Em estudo experimental, é apontado que um material ideal de fixação em osso necessita apresentar uma força, ao ser fixado, igual ou maior ao osso em questão e baixa perda dessa com o tempo. Além do tempo de absorção não causar risco de rachar ou formação de cistos, possibilidade de adquirir diversas formas inerente ao osso sem necessidade da remoção do mesmo. Além de fazer uma relação de algumas placas e por quê elas podem ter o risco de falha, como as PGAs e PLAs, por serem muito cristalinas e hidrofóbicas e a necessidade de uma fonte de calor para caso a moldagem prévia não esteja devidamente adequada a forma anatômica do indivíduo. Se aprofunda positivamente também na tecnologia de 
biocompostos reforçados para implantes onde a taxa do período de absorção chega a ser muito parecida a de placas de polímeros mas com o porém de ser mais fácil a osteogênese do organismo penetrando na placa, e de grande flexibilidade quando comparado com as demais, - que geram uma melhor degradação do composto e da formação de novo osso onde foi inserida, deixando uma maior integração no osso ao redor e preenchimento com formação óssea ${ }^{4}$.

Como uma alternativa minimamente invasiva, a redução aberta assistida por endoscopia em fixação interna fornece um bom campo visual e busca. É sabido que as placas de titânio geralmente são mais submetidas a esta técnica por conta da sua facilidade de biocompatibilidade. Porém, essas placas necessitam de remoção devido à sensação de corpo estranho ou à perda de aderência dos parafusos após a cicatrização das fraturas. Sob essa perspectiva, foi relatado que as placas biodegradáveis também são aplicáveis ao procedimento, além de fornecerem estabilidade pós-operatória na cicatrização das fraturas. As únicas complicações foram: inchaço leve, que se acredita ser causado por exercícios de abertura bucal 3 semanas após a cirurgia e dor nas articulações temporomandibulares ${ }^{20}$.

CONCLUSÃO

A partir dos estudos que foram analisados, pode-se inferir que a utilização da placa de titânio ainda é prevalente no quesito cirúrgico. Essa ocorrência se atribui ao custo mais acessível comparado às placas absorvíveis. Conquanto, foi possível constatar que 0 uso de placas biodegradáveis é aconselhado para uso pediátrico pois minimizam as restrições ao crescimento, dispensam a necessidade de uma cirurgia secundária, reduzindo, assim, gastos hospitalares. Além disso, as infecções associadas ao uso dos dois tipos de placas foram estatisticamente insignificantes. Diferente das placas de titânio, as placas biodegradáveis originam exames de imagem como RM com menos artefatos, de modo que não comprometa - diagnóstico de processos patológicos evoluídos para o câncer, como o carcinoma.

Sendo assim, as placas de biocomposto reforçado surgem como uma alternativa, haja vista que estas possuem grande flexibilidade e propriedades osteoindutoras na própria placa. Portanto, a tomada de decisão entre usar placas metálicas ou biodegradáveis deve abordar os aspectos citados neste estudo, e ser condicionada entre o cirurgião-dentista e o paciente, visando assegurar o bem-estar do indivíduo e a eficácia do tratamento.

\section{REFERÊNCIAS}

1. Laughlin RM, Block MS, Wilk R, Malloy RB, Kent JN. Resorbable plates for the fixation of mandibular fractures: a prospective study. J Oral Maxillofac Surg. 2007;65(1):89-96.

2. Paes JV, de Sá Paes FL, Valiati $R$, de Oliveira MG, Pagnoncelli RM. Retrospective study of prevalence of face fractures in southern Brazil. Indian J Dent Res. 2012;23(1):80-6.

3. Wu CM, Chen YA, Liao HT, Chen $\mathrm{CH}$, Pan $\mathrm{CH}$, Chen CT. Surgical treatment of isolated zygomatic fracture: Outcome comparison between titanium plate and bioabsorbable plate. Asian J Surg. 2018;41(4):370-376.

4. Baravarian $B$, Lindner TP, Merchav-Feuermann R. Advancements in Bone Fixation Utilizing Novel Biointegrative Fixation Technology. Clin Podiatr Med Surg. 2018;35(1):53-62.

5. Sukegawa $S$, Kanno $T$, Nagano D, Shibata $A$, Sukegawa-Takahashi Y, Furuki Y. The Clinical Feasibility of Newly Developed Thin Flat-Type Bioresorbable Osteosynthesis Devices for the Internal Fixation of Zygomatic Fractures: Is There a Difference in Healing Between Bioresorbable Materials and Titanium Osteosynthesis? J Craniofac Surg. 2016;27(8):2124-129.

6. Rha EY, Paik H, Byeon JH. Bioabsorbable plates and screws fixation in mandible fractures: clinical retrospective research during a 10-year period. Ann Plast Surg. 2015;74(4):432-36.

7. Yang RT, Lv K, Zhou HH, Li Z, Li ZB. Resorbable plates for the fixation of isolated mandibular angle fracture. J Craniofac Surg. 2015;26(2):447-48.

8. Moher D, Liberati A, Tetzlaff J, Altman DG; PRISMA Group. Preferred reporting items for systematic reviews and meta-analyses: the PRISMA statement. PLoS Med. 2009;6(7):e1000097.

9. Burlini D, Conti G, Amadori F, Bardellini E, De Giuli C. Management of paediatric maxillofacial fractures: conventional methods and resorbable materials. Eur J Paediatr Dent. 2015;16(1): 24-8.

10. Omezli MM, Torul D, Polat ME, Dayi E. Biomechanical comparison of osteosynthesis with poly-L-lactic acid and titanium screw in intracapsular condylar fracture fixation: An experimental study. Niger $\mathrm{J}$ Clin Pract. 2015;18(5):589-93.

11. An J, Jia $P$, Zhang $Y$, Gong $X$, Han $X$, He $Y$. Application of biodegradable plates for treating pediatric mandibular fractures. J Craniomaxillofac Surg. 2015;43(4):515-20.

12. Kim NK, Nam W, Kim HJ. Comparison of miniplates and biodegradable plates in 
reconstruction of the mandible with a fibular free flap. $\mathrm{Br} J$ Oral Maxillofac Surg. 2015;53(3):223-29.

13. Sakamoto $\mathrm{Y}$, Minabe $\mathrm{T}$, Kato $\mathrm{T}$, Kishi $\mathrm{K}$. Assessment of the RIVET fixation system for cranioplasty using the pull-out technique. J Craniomaxillofac Surg. 2015;43(2):281-84.

14. Oh JS, Kim SG. In vitro biomechanical evaluation of fixation methods of sagittal split ramus osteotomy in mandibular setback. J Craniomaxillofac Surg. 2015;43(2):186-91.

15. Konofaos P, Goubran S, Wallace RD. The Role of Resorbable Mesh as a Fixation Device in Craniosynostosis. J Craniofac Surg. 2016;27(1):105-8.

16. Mahmoud SM, Liao HT, Chen CT. Aesthetic and Functional Outcome of Zygomatic Fractures Fixation Comparison With Resorbable Versus Titanium Plates. Ann Plast Surg. 2016;76 Suppl 1:S85-90.

17. Leno MB, Liu SY, Chen CT, Liao HT. Comparison of functional outcomes and patientreported satisfaction between titanium and absorbable plates and screws for fixation of mandibular fractures: A one-year prospective study. J Craniomaxillofac Surg. 2017;45(5):7049.

18. Essig $H$, Lindhorst $D$, Gander $T$, Schumann $P$, Könü D, Altermatt $S$, Rücker M. Patient-specific biodegradable implant in pediatric craniofacial surgery. J Craniomaxillofac Surg. 2017;45(2):216-22.

19. Nguyen DC, Woo AS, Farber SJ, Skolnick GB, Yu J, Naidoo SD, Patel KB. Comparison of Resorbable Plating Systems: Complications During Degradation. J Craniofac Surg. 2017;28(1):88-92.

20.Son JH, Ha J, Cho YC, Sung IY. Are Biodegradable Plates Applicable in EndoscopeAssisted Open Reduction and Internal Fixation of Mandibular Subcondyle Fractures? J Oral Maxillofac Surg. 2017;75(8):1706-1715.

21. Chu SG, Lee JS, Lee JW, Yang JD, Chung HY, Cho BC, Choi KY. Comparisons among four types of absorbable plates used for internal fixation of zygomaticomaxillary complex fractures. J Craniomaxillofac Surg. 2019;47(3):383-88.

22. Na WG, Lim H, Koh SH. Three-Dimensional Computed Tomography Analysis of Stability Following Two- and Three-Point Fixation With Biodegradable Plates Among Patients With Zygomatic Fracture. J Craniofac Surg. 2019;30(2):478-82.

23. Song IS, Choi J, Kim SR, Lee JH. Stability of bioresorbable plates following reduction of mandibular body fracture: Three-dimensional analysis. J Craniomaxillofac Surg. 2019;47(11):1752-757.
24. Cural Ü, Atalay B, Yildirim MS. Comparison of Mechanical Stabilization of the Mandibular Angulus Fracture Fixation, With Titanium Plates and Screws, Resorbable Plates and Screws, and Bone Adhesives. J Craniofac Surg. 2018;29(7):1780-787.

25. Ueki K, Yoshizawa K, Moroi A, Hotta A, Tsutsui T, Fukaya K, Hiraide R, Takayama A, Tsunoda T, Saito Y, Baba N, Sato M. Modified hybrid fixation using absorbable plate and screw for mandibular advancement surgery. J Craniomaxillofac Surg. 2017;45(11):1788-793.

26. Janickova $M$, Statelova D, Mikuskova $K$, Jesenak M, Malachovsky I. Biodegradable versus titanium plates and screws for paediatric facial skeleton fractures. Bratisl Lek Listy. 2018;119(9):554-59.

27. Kim DY, Sung IY, Cho YC, Park EJ, Son JH. Bioabsorbable plates versus metal miniplate systems for use in endoscope-assisted open reduction and internal fixation of mandibular subcondylar fractures. J Craniomaxillofac Surg. 2018;46(3):413-17.

28. Filinte GT, Akan IM, Çardak GNA, Mutlu ÖÖ. Pediatrik mandibula kırıklarındaki ikilem: Eriyen plaklar mı yoksa metal plaklar mı? Ulus Travma Acil Cerrahi Derg. 2015; 21(6): 509-13.

29. Sukegawa $S$, Kanno T, Matsumoto $K$, Sukegawa-Takahashi Y, Masui M, Furuki Y. Complications of a poly-L-lactic acid and polyglycolic acid osteosynthesis device for internal fixation in maxillofacial surgery. Odontology. 2018;106(4):360-68.

30. Yan G, Chuo W, Zhang R, Zhou Q, Yang M. Evaluation of the Effect of Bioresorbable Plates and Screws in the Treatment of Condylar Fractures, Assisted by Digital Preoperative Planning. J Oral Maxillofac Surg. 2019 Jul;77(7):1434.e1-1434.e16.

31. Murakami K, Yamamoto K, Sugiura T, Horita S, Matsusue Y, Kirita T. Computed TomographyBased 3-Dimensional Finite Element Analyses of Various Types of Plates Placed for a Virtually Reduced Unilateral Condylar Fracture of the Mandible of a Patient. J Oral Maxillofac Surg. 2017;75(6):1239.e1-1239.e11.

32. Branch LG, Crantford C, Cunningham T, Bharti G, Thompson J, Couture D, David LR. LongTerm Outcomes of Pediatric Cranial Reconstruction Using Resorbable Plating Systems for the Treatment of Craniosynostosis. J Craniofac Surg. 2017;28(1):26-9.

33. Yasonov SA, Lopatin AV, Bel'chenko VA, Vasil'ev IG. Biodegradiruemye sistemy fiksatsii v detskol cherepno-litsevoĭ khirurgii: 10-letniI opyt ispol'zovaniia u 324 patsientov [Biodegradable fixation systems in pediatric craniofacial surgery: 10-year experience with 324 patients]. Zh Vopr Neirokhir Im N N Burdenko. 2017;81(6):48-55. 
34. Yan G, Zhang R, Chuo W, Gao X, Zhou Q, Yang M. Open Reduction Effects of Digitally Treating Zygomaticomaxillary Complex Fractures With Bio-Resorbable Materials. J Oral Maxillofac Surg. 2020 Jun;78(6):986-95.

35. Mukherjee CG, Mukherjee U. Maxillofacial trauma in children. Int $\mathrm{J}$ Clin Pediatr Dent. 2012;5(3):231-36.

36. Sim FW. Re: Kim NK, Nam W, Kim HJ. Comparison of miniplates and biodegradable plates in reconstruction of the mandible with a fibular free flap. $\mathrm{Br} \mathrm{J}$ Oral Maxillofac Surg. 2015;53(7):675.
CONFLITO DE INTERESSES

Os autores declaram não haver conflitos de interesse

AUTOR PARA CORRESPONDÊNCIA

\section{Georgia Costa de Araújo Souza}

Av. Rio Branco, 725 - Centro

59300-000 Caicó - RN, Brasil

E-mail:georgia_odonto@yahoo.com.br

Submetido em 25/02/2021

Aceito em 16/07/2021 